\title{
USING KNOWLEDGE MANAGEMENT TO IMPROVE QUALITY
}

\author{
Tilo Pfeifer, Reinhard Freudenberg $\left(^{*}\right)$, \\ Guido Hanel \\ Laboratory for Machine Tools and Production Engineering \\ Aachen University of Technology \\ R.Freudenberg@wzl.rwth-aachen.de \\ GERMANY
}

\begin{abstract}
Knowledge Management more and more gets a key element for the competitiveness of a lot of companies. In this connection Knowledge Management means several activities at different levels. This document presents the possibilty to use Knowledge Management to improve Quality. It will be shown that at some points "KM" and "QM" are close together.
\end{abstract}

\section{INTRODUCTION}

Where has the sudden interest in knowledge come from? Innumerable conferences and an abundance of contributions in specialist journals bear witness to the fact that knowledge and the associated science of knowledge management are the buzz words of the moment. The trend towards knowledge consultation and the projects on the subject of knowledge management currently under way in virtually all large companies, reflect the growing conviction that knowledge about knowledge is vital to the future success of the company. Numerous publications even assume that in the future, it will be the production factor and that it will be of decisive significance to the organisational survival of the company.

Yet is knowledge management really so fundamentally new or do we all apply some form of knowledge management? On closer examination of the knowledge management models, we find numerous elements which are already used in some form or another in quality management. This presents the opportunity to enhance the quality of quality management by identifying these elements.

In this presentation, we shall move from general thoughts on knowledge management to looking at how elements of knowledge management can be integrated with quality management to develop a knowledge-based quality management system. 


\section{THE NEED TO MANAGE KNOWLEDGE}

Thirty companies known to be interested in knowledge management, were surveyed in the period from July to November 1998by the Faculty of Economics at the Polytechnic in Wiesbaden, led by Prof. Dr.-Ing. Klaus North. The majority of these companies were large companies with over 1,000 employees and most of them were based in the service sector (North, 1998).

Many of these companies were and remain uncertain as to why they should apply knowledge management. Is the purpose of engaging in knowledge management solely to demonstrate publicly that the company is "in with the best of them" or is there a genuine and more pressing reason?

It emerged, that over a quarter of companies were addressing this subject because it is the subject of intensive public discussion. The sources of information quoted, were primarily congresses, publications in specialist journals and new publications in the specialist literature (see figure 1).

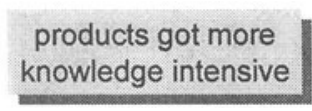

\section{improvement of the internal knowledge structure and-exchange}
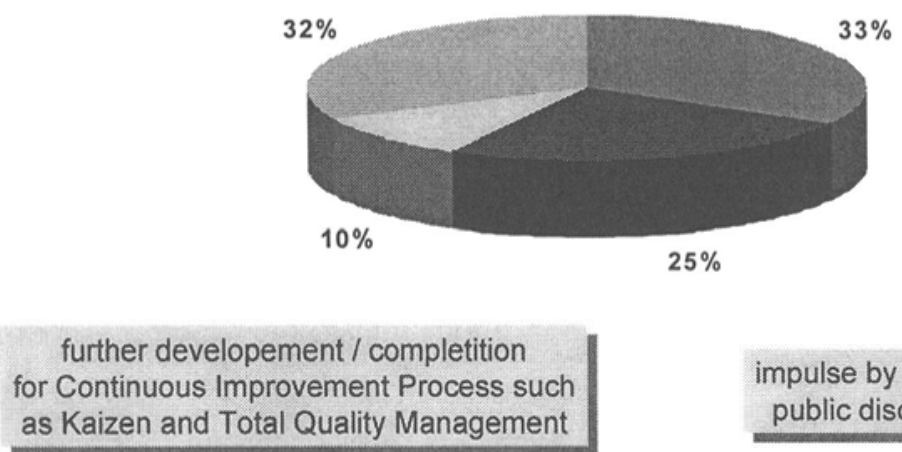

\section{impulse by intensive public discussion}

Figure 1 - Why Knowledge Management

However, the majority of the companies questioned said that they had other reasons. One third of the companies hoped to apply knowledge management techniques to improve internal knowledge development and transfer. One of the reasons for this is the increased level of globalisation of company activity and the increase in the size of the company. It is essential, therefore, to ensure not only that a large number of employees is given access to the knowledge required, the knowledge must also be updated and made available in its entirety to all of the company's bases, many of which are distributed worldwide.

A further third quoted the fact that the products have become more knowledge intensive, as a reason for addressing knowledge management. This is reflected in the fact that it is no longer sufficient for companies to offer only "products" but are increasingly being forces to supply "total solutions", if they wish to remain competitive. In such a situation, the old sales and marketing structures cease to be 
efficient since inter-departmental expertise is frequently required in order to produce the solutions required.

$10 \%$ of the companies questioned, regard the application of knowledge management as offering a direct opportunity to optimise established techniques such as Kaizen and Total Quality Management.

The study shows that there are a number of reasons for companies to investigate the opportunities presented by knowledge management techniques.

But is there any one universally valid reason why it should be necessary to apply knowledge management? Are there any indicators which flag up the need for the application of knowledge management techniques? A few examples of such indicators are outlined in the following.

When the same faults occur repeatedly in a company, this shows that there are gaps in the knowledge of the employees about processes and operations. Equally, it is not unknown, particularly in large companies, for various departments to be working in isolation on the same problem, when none of the employees in the departments involved, is aware that other departments are doing the same thing. In such situations, there is considerable potential for improvement (the frequently quoted saying "If Siemens knew what Siemens knows" springs to mind).

In the DaimlerChrysler AG, for example, over 100 million DM must be set aside each year in to cover guarantee commitments and goodwill payments for complaints which are attributable to known design faults. It is not difficult to imagine the significance of this problem in terms of the whole national economy. The German Economics Institute has estimated that the annual damage to industry incurred by the repeated development of solutions which were already known, amounts to several thousands of millions of D-Marks.

It is particularly dangerous when the company is reliant on certain key personnel, who are the holders of "central reserves" of knowledge. In the currently prevailing atmosphere of cut-throat competition which prevails, particularly in the area of IT, a situation like this can put the existence of the entire company at risk when key personnel choose to leave and work for the competition. This hidden knowledge must be made accessible to the company as a whole.

It is also important to keep an eye on the situation with regard to the development of new products and services - as well as of corporate structures. Where the progress of new developments is sluggish, the selective application of knowledge management may provide the remedy (Bonaventura, 1997).

\section{THE WAY TO MANAGE KNOWLEDGE}

But what exactly is knowledge management? It can be described most succinctly, using Probst's Knowledge Management Circulation. In his works, Probst identified six important steps and united them in a closed circulation (Probst, 1999). It is important to note at this point that there is absolutely no need to carry out these steps sequentially and that each of them can also be applied in isolation (see figure 2).

These are described briefly in the following:

One of the principal elements in knowledge management is the identification of knowledge which is already available. The objective during this phase, is to clarify the level of internal and external knowledge. Similarly, any gaps in knowledge and 
in skills are revealed in this process. This knowledge transparency gives the individual in the organisation a better sense of orientation and more efficient access to internal and external knowledge. Examples of this include knowledge topographies, knowledge maps and expert networks.

The next phase consists of the acquisition of knowledge. In this phase, the question arises as to how external knowledge ( i.e. the knowledge which exists outside the company) can be accessed. Possible means of achieving this include the acquisition of the knowledge of external knowledge holders (personnel recruitment, consulting projects), the acquisition of stakeholder-knowledge (e.g. customers, suppliers), the acquisition of the knowledge of other companies (collaborative projects) and the acquisition of knowledge products (software, databases, patents, licence agreements, development contracts).

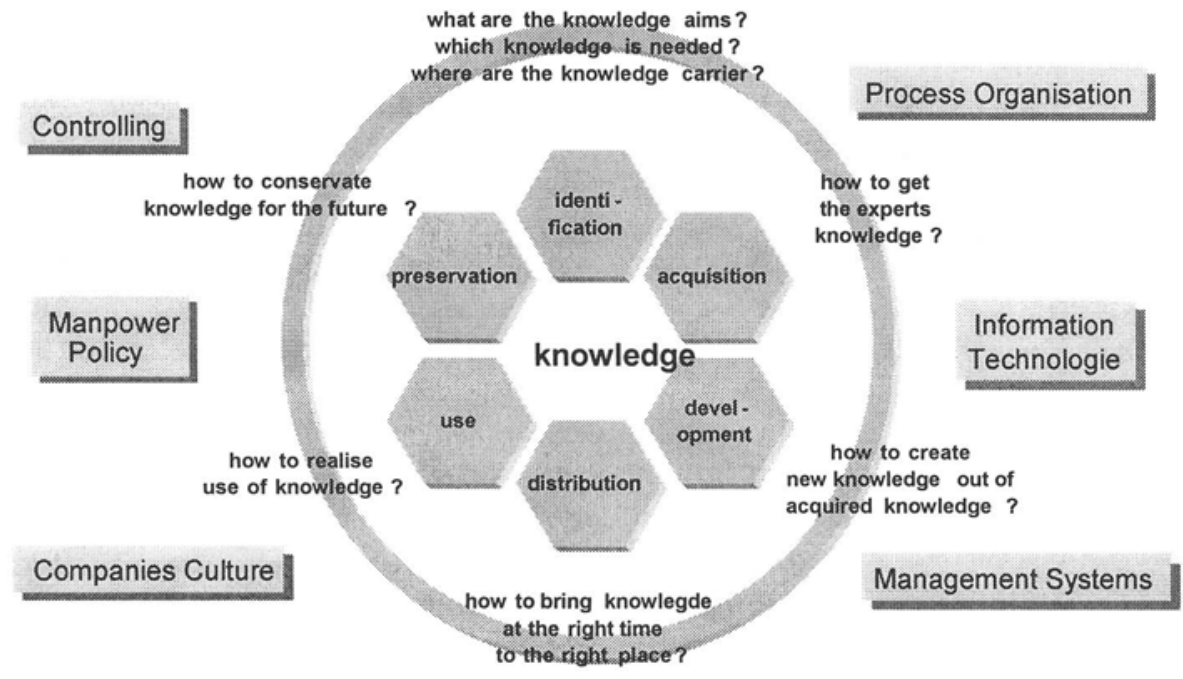

Figure 2 - Modules of Knowledge Management

The knowledge development phase examines the question as to how the knowledge acquired can be used to create new knowledge. Here, a distinction can be drawn between individual and collective learning processes (e.g. „lessons learned“). In this context, the role of knowledge management is to create a fertile environment.

The knowledge which is available must be distributed. This phase, therefore, addresses the question as to how knowledge can be made available at the right place at the right time. So the aim must be to channel islands of information and knowledge held by individuals, to stocks of knowledge which are centrally available. Here, distinctions can be drawn between the central dissemination of collective knowledge to specific groups of employees (knowledge multiplicators, push strategy) or the decentralised imparting or sharing of knowledge among individual employees, working groups or teams (knowledge networks; pull strategy). 
The primary aim of all knowledge management activities, is certainly to ensure that knowledge is utilised. The term utilisation, in this context, refers to the productive deployment of organisational knowledge, i.e. the transfer of knowledge into action and decision-making. The function of quality management is to create the conditions within companies which will ensure that knowledge which is either already available, acquired or developed, is actually utilised. Knowledge must come to be regarded as a resource, which must be used to ensure the general benefit of the company, regardless of its source. On an individual level, this calls for a willingness to continuously ask whether established sequences still make sense and for the prevention of any occurrence of the "not-invented-here-syndrome" (attitude which rejects any solutions which were not developed within the department or company involved). Davenport uses the succinct term "knowledge market" to describe the environment which must be developed within a company. The mechanisms and, according to Davenport, the principles on which this knowledge market is based, are the same as those in the conventional market (customers, buyers, supply, demand "price of knowledge", ).

Ultimately, the question arises as to how knowledge can be preserved for the future of the company. Here, it has emerged that the measures which have been popular in recent years, for example Business Process Reengineering or Outsourcing have resulted, in many cases in a frequently irreversible loss of individual and collective knowledge, and consequently, in a reduced level of corporate efficiency. Here, ICT for example, can make a contribution by recording and preserving knowledge in knowledge documents such as knowledge maps or "lessons learned".

Where can the knowledge circulation be used?

On closer examination, these knowledge management modules can play a supporting role by improving key processes in questions relating to the subjects including the organisation of processes, the development of a productive corporate culture, personnel management, controlling modules and thus in the organisational structure.

The previously mentioned study undertaken by the Polytechnic University in Wiesbaden, revealed that the potential of knowledge management to enhance organisational structures has achieved recognition. The companies were asked what type of measures they are currently implementing or intend to implement in the future. A distinction was drawn between organisational measures, personnel measures and measures in the field of information and communications technology. It emerged that the majority of the companies $(41 \%)$, had chosen to implement a combination of ICT and organisational measures. A total of $72 \%$ of all companies questioned reported that their focus in knowledge-management activities was on the organisational aspects.

The focus of this presentation will therefore also be on organisational questions.

The strong emphasis on organisational measures raises the question as to the aim which is being pursued. Too frequently, the introduction of a knowledge management concept is equated within the company, with that of a computer system or of new software. The company management or the advocates of the knowledge management system within the company expect only that staff will use the correct communications software to communicate their knowledge and that the knowledge market referred to earlier, will thus become a vibrant entity. In fact, any management which does this is actually putting the carriage in front of the horse. 
Thorough preparation is essential before the opportunities presented by ICT can be fully accepted and exploited.

This preparation is reflected in the knowledge management in the "right" corporate culture. In many projects of this nature, it is forgotten that only a fraction of the corporate knowledge is available in document form and can thus be processed directly by the computer systems. Numerous studies assume that over $2 / 3$ of the knowledge in companies remains in the brains of the employees (N.N., 2000). Consequently, the first and most fundamental task in the management of knowledge is to encourage staff to share their knowledge, to communicate. Only then can the advantages of rapid non-location bound distribution via ICT be exploited to the full.

A knowledge-oriented corporate culture can be described by four principles. In order of importance, these are: Trust, reciprocity, reputation and selflessness (Davenport, 1999).

Trust is the vital prerequisite and the basis for any dissemination of knowledge. Top management must exemplify this attitude. Any company management which publicly proclaims its support for information exchange and sharing but which actually rewards staff for keeping their knowledge to themselves, will never be able to establish an effective system of knowledge management.

The willingness to share information, also depends on the principle of reciprocity. Any employee who share their knowledge with a colleague, expect reciprocal action either directly or in the future. Employees who willingly share information expect their colleagues to be equally ready to share. It is essential to ensure that there is a strong culture of partnership within the company.

Reputation is closely associated with this. Employees who are known to share their knowledge readily, can expect to be able to call on reciprocal action more easily. It is vital in this context, that the knowledge provided, is always linked to the name of the supplier. In groupware systems, for example, it is important to ensure that the appropriate mechanisms are in place to register both the use and the supplier of the information entered. At this point, material advantages can be set up in order to further reinforce the knowledge-oriented corporate culture. Employees who have a reputation for their willingness to share information should be rewarded by increased workplace security, promotion or by the payment of bonuses.

The fourth and last characteristic, can be described as selflessness. This characteristic cannot be created let alone expected; it can only be encouraged and promoted. Mentor programs, for example, are based on this type of willingness to help. Experienced staff with the corresponding areas of expert knowledge can be encouraged to act as mentors for new staff. It is essential that the company management recognise this function officially and release sufficient resources for it to be performed efficiently. These resources must include sufficient time and a suitable place.

It is important to note that the two last points are not restricted to the upper echelons of the corporate culture but must be accorded a position of central importance in all areas of knowledge management activities. Each member of staff must be given the time to acquire, generate and disseminate knowledge (Malhotra, 1998). 


\section{LINKS BETWEEN QUALITY AND KNOWLEDGE MANAGEMENT}

The modules which form knowledge management and the importance of addressing organisational questions, have now been outlined.

An examination of the significance of quality and knowledge management for the company, reveals that in economic terms, the success of a company depends on two types of growth. Firstly, companies must maximise profits and secondly, they must increase their share of the market. This pressure for success is intensified by increased globalisation with ever more transparent and rapidly moving markets as well as by the demands of the stakeholders.

A process-oriented view of company sequences of operations currently offers the best means of increasing the efficiency of the entire value-added process. This gives rise to demands for an efficient, process-oriented quality management system. This approach can be regarded as a management function which is applied in order to ensure a continuous increase in effectiveness as a result of products which have been adapted to meet the requirements of specific customers and the continuous increase in efficiency by avoiding superfluous or the wrong operations from being carried out. Two important considerations in this context are:

- The guarantee of suitable and continuously updated company aims (Required Quality) and

- The provision of optimum translation of targets into product and process efficiency (Process Quality).

The employees occupy a position of central importance not only in assuring quality from the points of view of "targets" and "processes" but also in the conventional sense of product quality. The must be informed, motivated and trained via an efficient and successful knowledge management system in order to enable them to produce the required levels of achievement and quality in all areas in which they are involved.

It makes sense, therefore, to examine these two management approaches as one unit, i.e. not in isolation from one another. The benefits of this approach have been verified by a study conducted by the Fraunhofer-Institute IAO in Stuttgart. The most frequently expressed expectation of knowledge management is "enhanced product quality". Expectation of "increased capacity for innovation" and "increased customer orientation" reached positions 2 and 3 respectively. Quality managers and knowledge managers are therefore pursuing the same aims and are indeed like runners on parallel sometimes overlapping tracks. This is taken into account in the revised edition of the Quality Management System Standards ISO 9000/2000 and by the "Model for Business Excellence" of the European Foundation for Quality Management. The emphasis in the following will therefore be on the changes to the revised 2000 edition of the ISO 9000-series and in the EFQM model.

DIN EN ISO 9004 explicitly demands the availability of information needed to conduct and monitor processes. The organisation was therefore given the task of identifying suitable sources of information and of ensuring timely access to the information.

Even when knowledge management is not mentioned by name at any point, the term "information" can replaced throughout by "knowledge" and numerous parallels 
to the knowledge management circulation are apparent. In order to justify this, it makes sense at this point, to define the terms knowledge and information. It is knowledge which enables humans to make decisions, to act and to solve problems; i.e. also to pursue strategies and reach targets. Information is simply the raw material which generates knowledge.

The formulation in the current EFQM model, is different, but can easily be transferred to knowledge management.

In April 1999, The EFQM presented an altered EFQM-Model, which was the subject of discussion. The name was changed to the "Model for Business Excellence" in order to express more strongly the fact that it is an integrative model for evaluating companies (see figure 3 )

One of the principal changes, is the addition of further elements to complete the model, forming a control loop. The division into "facilitators" and "results" is represented by an arrow above the criteria. Both terms are completed by the word "process" in order to emphasise the need for process thinking. Thus the new EFQM model focuses attention even more strongly on the significance of process-oriented quality management approaches .

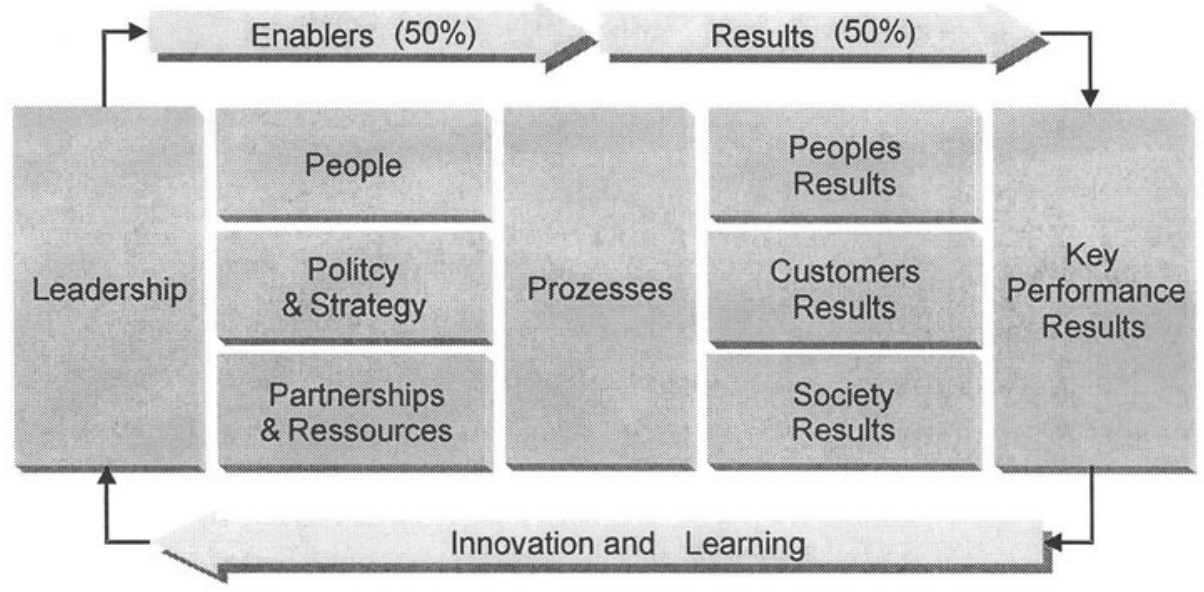

Figure 3 - The new EFQM-Model

The control loop is closed by an arrow below the criteria containing the terms "innovations" and "learning". By highlighting "Learning" which was previously related only to the term employee, it is now applied to the entire model. This emphasises that learning must move beyond the learning of the individual and must involve the whole organisation in a learning process. In conjunction with innovations, this, in turn, should ensure a cycle of improvement in the application of the model and in its implementation in the company. 


\section{KNOWLEDGE BASED QUALITY MANAGEMENT}

The brief outline of the new DIN EN ISO 9000 and the EFQM-Model demonstrate again that knowledge management and quality management are linked. When this link is analysed in depth, three principle approaches emerge.

Firstly, the quality of the knowledge should be increased and assured systematically. Existing methods and approaches used in quality management can be used to ensure that the quality of knowledge is comparable to the quality, for example of the products. To achieve this, criteria permitting the knowledge in the company to be evaluated qualitatively must be developed. These criteria already exist and are applied in the field of information. The information content, or the usefulness of the information, for example, can be used as a measure of the quality of information. Until now, there has been a lack of comparable criteria which can be applied to knowledge.

Secondly, the knowledge management processes should be secured systematically. This can be achieved by dealing consciously with the resource knowledge in defined processes. A comprehensive quality management concept can be drawn up, when these two factors are given adequate consideration.

The last linking approach focuses on the development of knowledge management systems which highlight the knowledge perspective within the company and which can be integrated easily within existing management systems. In terms of quality management systems, this means that the knowledge required to perform and check processes, must be made available (Pfeifer, 2000).

What does the availability of knowledge mean to companies today? A successful and efficient knowledge management system will have to do more than simply ensure that the available knowledge is communicated at all. It is likely that the efficiency of the knowledge handling process will depend on the speed at which knowledge can be distributed. Companies which operate globally must guarantee this speed for all of their bases, taking account of the individual requirements of the employees. The approaches adopted in the fields of global engineering and the burgeoning E-commerce, show that companies will have to cope with these demands first in the areas of product and service development as well as in Sales and Services. The so-called "247" development projects (projects which run 24 hours per day, 7 days per week over all time zones) will be required to use a new form of project knowledge. Designers in Asia will have to be able to rely on the experience of European staff, unimpeded by time differences. Companies will be faced with similar challenges throughout the entire area of E-commerce. The contact with customers, reduced to e-mail or internet communications will call for new solutions which must ensure that valuable customer knowledge is passed back to the company.

Quality management will play a vital role in all of these new challenges. The objective for the optimisation of quality management techniques will be to secure product and process quality by ensuring the availability of the appropriate knowledge where and when it is needed in knowledge intensive business processes. 


\section{REFERENCES}

1. Bonaventura M. "The Benefits of a knowledge culture.". Aslib Proceedings, 49(4): 82-89, April 1997.

2. Davenport Th, Prusak L. Das Praxisbuch zum Wissensmanagement 1998, Verlag Moderne Industrie, Landsberg/ Lech

3. Malhotra Y. "Toward a Knowledge Ecology for Organizational White-Waters" Keynote Presentation at the Knowledge Ecology Fair 98: Beyond Knowledge Management, Feb. 2 - Feb. 27, 1998,

4. N.N. Wissensmanagement-ein wichtiger Teil ist die Überzeugung der Mitarbeiter. VDMA Maschinenbau-Nachrichten, Nr.02, 2000

5. North K, Papp A. Erfahrungen bei der Einfuhrung von Wissensmanagement, Warum und wie Unternehmen das Neuland Wissensmanagement erobern. io-management, Nr.4, 1999

6. Pfeifer T; Scheermesser S, Lorenzi P. Verborgene Potenziale erschließen; Wissensmanagement und Qualitătsmanagement- eine Erfolg versprechende Verbindung? QZ, Jahrgang 45(2000)3, 2000

7. Probst G, Raub S, Romhardt K. Wissen managen- Wie Unternehmen ihre wertvollste Ressource optimal nutzen. 3. Auflage, 1999, Gabler Verlag, Frankfurt a.M. 